\title{
PERSISTENCE DEFINITIONS AND THEIR CONNECTIONS
}

\author{
H. I. FREEDMAN AND P. MOSON \\ (Communicated by Kenneth R. Meyer)
}

\begin{abstract}
We give various definitions of types of persistence of a dynamical system and establish a hierarchy among them by proving implications and demonstrating counterexamples. Under appropriate conditions, we show that several of the definitions are equivalent.
\end{abstract}

\section{INTRODUCTION}

The main purpose of this paper is to consider various forms of persistence (defined in the next section) in dynamical systems and to establish a hierarchy among them.

The work in this paper may be thought of as a continuation of work done in [2] (also see [3]). In [2], the concepts of weak persistence, persistence and uniform persistence were defined for the first time for dynamical systems in a locally compact metric space with respect to sets with boundary and nonempty interior. From the definitions it was clear that uniform persistence implies persistence, which in turn implies weak persistence. It was then shown that, under certain circumstances, weak persistence implies uniform persistence.

In this paper, we define two additional concepts of persistence, one which is weaker than weak persistence, denoted $W$-weak persistence, and has been used in applications, and the other which is a uniform version of weak persistence, denoted weak uniform persistence. Among other results, we prove that if the flow is dissipative or if the boundary is compact, then weak uniform persistence is equivalent to uniform persistence.

Previous work in persistence theory has typically dealt with the abstract theory, with applications, or with both. Criteria for the equivalence of various forms of persistence were considered in [2,3]. Criteria for one or more forms

Received by the editors April 18, 1989 and, in revised form, August 28, 1989.

1980 Mathematics Subject Classification (1985 Revision). Primary 34C35; Secondary 58F25, $92 \mathrm{~A} 15$.

The first author's research was partially supported by the Natural Sciences and Engineering Research Council of Canada, Grant No. NSERC A4823.

The second author's research was partially supported by the Hungarian National Foundation for Scientific Research and was carried out while the author was a WUSC research associate at the University of Alberta. 
of persistence to hold in general dynamical systems were given in $[3,4,7,10,11$, $13,18,19]$, in Lotka-Volterra systems in $[10,11,13,14]$, in infinite dimensional systems in $[1,9]$. Persistence theory as applied to population survival or extinction was discussed in $[5,6,12,14,15,18]$. There are also many papers dealing with persistence theory exclusively for discrete dynamical and semi-dynamical systems which are cited in the above references.

In a theoretical sense persistence definitions can be interpreted as a complete instability or as boundedness-like qualitative concepts (see [17, Chapter VI]). Our theorem about the equivalence of weak uniform persistence to uniform persistence is a modification of a theorem by V. A. Pliss [16] stating the equivalence of weak ultimate boundedness and ultimate boundedness for the case of ordinary differential equations with periodic coefficients.

The paper is organized as follows. In $\S 2$ we formulate the main definitions and notations. Section 3 is devoted to the connection between weak uniform persistence and uniform persistence. In $\S 4$ we present some counterexamples proving the independence of these persistence definitions. Finally in $\S 5$ we consider the special cases of autonomous and periodic Kolmogorov systems in $R^{n}$.

\section{Definitions AND NOtATIONS}

Here we briefly recall the definitions and notation introduced in [2], and define some new concepts as well.

Let $\varepsilon$ be a locally compact metric space, and $E=\bar{E}$ (the closure of $E$ ) $\subset \varepsilon$. We consider the continuous flow $\mathscr{F}=(E, R, \pi)$, where $\pi: E \times R \rightarrow E$, $\pi(\pi(x, t), s)=\pi(x, t+s), \forall x \in E, \forall s, t \in R$. To avoid trivialities assume that neither $\stackrel{\circ}{E}$ (the interior of $E$ ) nor $\partial E$ (the boundary of $E$ ) is empty. We call the flow $\mathscr{F}$ dissipative if $\forall x \in E$ the $\omega$-limit set $\Lambda^{+}(x) \neq \phi$ and $\Omega(\mathscr{F})=\bigcup_{x \in E} \Lambda^{+}(x)$ has compact closure. We shall say that

(i) $\mathscr{F}$ is weakly persistent (WP) if $\forall x \in \stackrel{\circ}{E}$,

$$
\limsup _{t \rightarrow+\infty} d(\pi(x, t), \partial E)>0,
$$

(ii) $\mathscr{F}$ is (strongly) persistent $(P)$ if $\forall x \in \stackrel{\circ}{E}$,

$$
\liminf _{t \rightarrow+\infty} d(\pi(x, t), \partial E)>0,
$$

(iii) $\mathscr{F}$ is weakly uniformly persistent (WUP) if $\exists \varepsilon_{0}>0 \ni \forall x \in \stackrel{\circ}{E}$,

$$
\limsup _{t \rightarrow+\infty}(d(\pi(x, t)), \partial E)>\varepsilon_{0} .
$$

(iv) $\mathscr{F}$ is uniformly persistent (UP) if $\exists \varepsilon_{0}>0 \ni \forall x \in \stackrel{\circ}{E}$,

$$
\liminf _{t \rightarrow+\infty}(d(\pi(x, t)), \partial E)>\varepsilon_{0} .
$$


The following obvious relations are valid for these definitions:

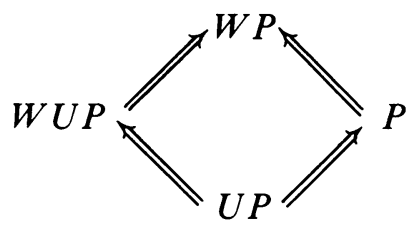

A nonempty invariant set for a flow which is the maximal invariant set in some neighbourhood of itself is called an isolated invariant set. Let $\partial \mathscr{F}$ be the restriction of $\mathscr{F}$ to $\partial E$ (assumed invariant under $\mathscr{F}$ ). The flow $\partial \mathscr{F}$ is isolated if there exists a finite covering $\mathscr{M}=\left\{M_{i}\right\}$ of $\Omega(\partial \mathscr{F})$ by pairwise disjoint compact isolated invariant sets $M_{1}, \ldots, M_{k}$ for $\partial \mathscr{F}$ such that each $M_{i}$ is also isolated for $\mathscr{F}$. Let $M, N$ be isolated invariant sets for $\mathscr{F}$. We shall say that $M$ is chained to $N(M \rightarrow N)$ if there exists $x \notin M \cup N$ such that the $\alpha$ and $\omega$-limit sets of $x$ belong correspondingly to $M$ and $N$. A chain of isolated invariant sets $M_{1} \rightarrow M_{2} \rightarrow \cdots \rightarrow M_{k}$ is a cycle if $M_{1}=M_{k}$. Finally $\partial \mathscr{F}$ is called acyclic if, for some isolated covering $\mathscr{M}$ of $\Omega(\partial \mathscr{F})$, no subset of $\mathscr{M}=\left\{M_{i}\right\}$ forms a cycle.

\section{CRITERIA FOR THE EQUivalence OF WUP AND UP}

In the previous section, we have introduced a new concept, namely weak uniform persistence. As mentioned there, $U P \Rightarrow W U P$. Here we state two theorems showing when $W U P \Rightarrow U P$.

Theorem 1. Let $\mathscr{F}$ be a continuous, dissipative flow on $E$ with metric $d$. Let $\partial \mathscr{F}$ be the restriction of $\mathscr{F}$ to $\partial E$ (assumed invariant under $\mathscr{F}$ ). Then $W U P \Rightarrow U P$.

Proof. Suppose not. Then there exist sequences $\left\{\tilde{x}_{k}\right\}_{k=1}^{\infty},\left\{e_{k}\right\}_{k=1}^{\infty}, \tilde{x}_{k} \in \stackrel{\circ}{E}$, $0<e_{k} \in R \ni \lim _{k \rightarrow+\infty} e_{k}=0$, and such that

$$
\liminf _{t \rightarrow+\infty} d\left(\pi\left(\tilde{x}_{k}, t\right), \partial E\right)<e_{k} .
$$

One can assume $e_{k}<\varepsilon_{0} \forall k$, where $\varepsilon_{0}$ is from the definition of $W U P$. that

From $W U P$ it follows that there exists a sequence $\left\{\tau_{k}\right\}_{k=1}^{\infty}, \tau_{k}>0$ such

$$
d\left(\pi\left(\tilde{x}_{k}, \tau_{k}\right), \partial E\right)>\varepsilon_{0} .
$$

As $\overline{\Omega(\mathscr{F})}$, the closure of the union of all $w$-limit sets, is compact and $E$ is locally compact, we can find an open set $G$ such that $\overline{\Omega(\mathscr{F})} \subset G$ and $\bar{G}$ is compact. Assume that $\tau_{k}$ is chosen large enough in the sense that if $t \geq \tau_{k}$, then $\pi\left(\tilde{x}_{k}, t\right) \in \bar{G}$.

From (1) it can be seen that there exists a sequence $\left\{\tilde{t}_{k}\right\}_{k=1}^{\infty}, \tau_{k}<\tilde{t}_{k}$ and that

$$
d\left(\pi\left(\tilde{x}_{k}, \tilde{t}_{k}\right), \partial E\right)<e_{k}
$$


Since $\mathscr{F}$ is continuous there exists a sequence $\left\{\tilde{\theta}_{k}\right\}$ such that $\tilde{\theta}_{k} \in\left[\tau_{k}, \tilde{t}_{k}\right]$ and

$$
d\left(\pi\left(\tilde{x}_{k}, \tilde{\theta}_{k}\right), \partial E\right)=\varepsilon_{0}
$$

and, for $\tilde{\theta}_{k}<t<\tilde{t}_{k}$,

$$
d\left(\pi\left(\tilde{x}_{k}, t\right), \partial E\right)<\varepsilon_{0} .
$$

Let $x_{k}=\pi\left(\tilde{x}_{k}, \tilde{\theta}_{k}\right)$. From the choice of $\tau_{k}$ and $\tilde{\theta}_{k}$ it is clear that $x_{k} \in \bar{G}$, which is a compact set, so we can assume that $\left\{x_{k}\right\}_{k=1}^{\infty}$ is a convergent sequence (otherwise we would choose a convergent subsequence). Let $\lim _{k \rightarrow+\infty} x_{k}=x^{\prime} \in$ $\bar{G}$. It follows from (4) that $d\left(x^{\prime}, \partial E\right)=\varepsilon_{0}$, so $x^{\prime} \in \stackrel{\circ}{E}$.

Let $t_{k}=\tilde{t}_{k}-\tilde{\theta}_{k}$. From the group property of $\pi$ and (3), (5), it follows that

$$
d\left(\pi\left(x_{k}, t_{k}\right), \partial E\right)<e_{k}\left(<\varepsilon_{0}\right)
$$

and, if $0<t \leq t_{k}$,

$$
d\left(\pi\left(x_{k}, t\right), \partial E\right)<\varepsilon_{0} .
$$

Now consider the orbit with initial point $x^{\prime}$. It follows from $W U P$ that there exists $\tau^{\prime}>0$ such that

$$
d\left(\pi\left(x^{\prime}, \tau^{\prime}\right), \partial E\right)>\varepsilon_{0} .
$$

Since $\stackrel{\circ}{E}$ is invariant under $\mathscr{F}$ we can find $0<m<\varepsilon_{0}$ such that

$$
d\left(\pi\left(x^{\prime}, t\right), \partial E\right)>m, \quad 0 \leq t \leq \tau^{\prime} .
$$

It follows from the continuity of $\pi, \lim _{k \rightarrow+\infty} x_{k}=x^{\prime}$, and (8), (9) that if $k$ is sufficiently large then

$$
d\left(\pi\left(x_{k}, \tau^{\prime}\right), \partial E\right)>\varepsilon_{0}
$$

and

$$
d\left(\pi\left(x_{k}, t\right), \partial E\right)>m\left(>e_{k}\right), \quad 0 \leq t \leq \tau^{\prime} .
$$

Inequalities (10), (11) contradict (6), (7). In fact if $\tau^{\prime}<t_{k}$, then (10) contradicts (7), if $\tau^{\prime} \geq t_{k}$ then (11) contradicts (6). These contradictions prove the theorem!

Theorem 2. In Theorem 1, if the dissipativity hypothesis is replaced by a hypothesis that $\partial E$ is compact, then again $W U P \Rightarrow U P$.

Proof. The existence of a convergent sequence $\left\{x_{k}\right\} \subset \bar{H}=\{x \mid x \in E, d(x, \partial E)$ $\left.\leq \varepsilon_{0}\right\}$ follows from the compactness of $\partial E$. Using $H$ instead of $G$, the proof follows as in Theorem 1 .

Corollary 3. If there exists $\varepsilon_{0}>0 \ni$ for all $x \in \stackrel{\circ}{E}, \exists \tau(x)>0 \ni d(\pi(x, \tau(x)), \partial E)$ $>\varepsilon_{0}$ then $\mathscr{F}$ is weakly uniformly persistent with constant $\varepsilon_{0}$.

Proof. Suppose not. Then there is an $\tilde{x} \in \stackrel{\circ}{E} \ni \lim \sup _{t \rightarrow+\infty} d(\pi(\tilde{x}, t), \partial E)=$ $\varepsilon_{1}<\varepsilon_{0}$. But then, for all sufficiently large $t \geq T, d(\pi(\tilde{x}, t), \partial E)<\varepsilon_{0}$. Let 
$x=x(\tilde{x}, T)$. From the group property of $\pi$ and the previous inequality it follows that

$$
d(\pi(x, t), \partial E)<\varepsilon_{0} \quad \forall t \geq 0,
$$

which contradicts the assumption of the statement.

Remark 4. Without additional assumptions, $W U P$ is not equivalent to $U P$. To show this, let $E=R_{+}^{2}$, and $\mathscr{F}$ the flow generated by the simplest LotkaVolterra system $\dot{x}=x(a-b y), \dot{y}=y(-c+d x), a, b, c, d>0$. All interior orbits are closed around the fixed point $\left(\frac{a}{b}, \frac{c}{d}\right)$, and, for any $\varepsilon_{0}, 0<\varepsilon_{0}<$ $\min \left(\frac{a}{b}, \frac{c}{d}\right), \mathscr{F}$ is $W U P$, but not $U P$.

\section{EQUiVALENCES AND NONEQUIVALENCES OF PERSISTENCE DEFINITIONS}

As already noted the following implications hold: $U P \Rightarrow W U P \Rightarrow W P$ and $U P \Rightarrow P \Rightarrow W P$. In the previous section we have shown that if the flow is dissipative, then $W U P \Rightarrow U P$, i.e., $U P$ and $W U P$ are equivalent. In this case one has $W U P=U P \Rightarrow P \Rightarrow W P$. Here we show that, even with dissipativity, $P \nRightarrow U P$ and $W P \nRightarrow P$. However, if certain additional assumptions (acyclicity, isolatedness of $\partial \mathscr{F}$ ) are made, then we note (see [2]) that $W P \Rightarrow U P$ so that all definitions are equivalent.

Example $5(W P \nRightarrow P)$. A counterexample demonstrating the trajectories of a dissipative, weakly persistent, but not persistent, system can be seen in Figure 1. The detailed description of the system is described below (see Figure 1). Let $E=R_{+}^{2}, \mathscr{F}$ be the flow generated by the system

$$
\dot{x}_{i}=x_{i} f_{i}\left(x_{1}, x_{2}\right), \quad i=1,2 .
$$

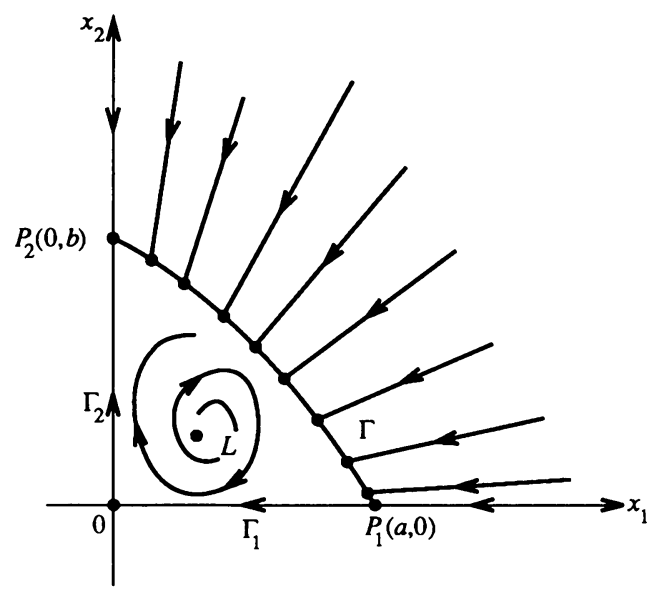

FIGURE 1. 
To obtain the required $f_{i}\left(x_{1}, x_{2}\right)$, we first consider the system

$$
\dot{x}_{i}=x_{i} g_{i}\left(x_{1}, x_{2}\right) \text {, }
$$

which has the following properties:

(i) There are four equilibria in $\mathbf{R}_{+}^{2}, O(0,0), P_{1}(a, 0), P_{2}(0, b), L\left(l_{1}, l_{2}\right)$, $a, b, l_{1}, l_{2}>0$.

(ii) $O, P_{1}, P_{2}$ are saddle points connected by separatrices as follows: $\Gamma_{1}$ denotes the orbit along the $x_{1}$ axis from $O$ to $P_{1}, \Gamma_{2}$ denotes the orbit along the $x_{2}$ axis from $P_{2}$ to $O, \Gamma$ denotes the orbit lying in $\stackrel{\circ}{R}_{+}^{2}$ from $P_{1}$ to $P_{2}$. We assume that the curve $\Gamma$ can be parametrized by the relation $x_{2}=\varphi\left(x_{1}\right), 0<x_{1}<a$. We then smoothly extend $\varphi$ on $\mathbf{R}^{+}$so that $\varphi\left(x_{1}\right)<0$ for $x_{1}>a$. We denote the open region bounded by $\bar{\Gamma}_{1}, \bar{\Gamma}_{2}$ and $\bar{\Gamma}$ as $D$, i.e.,

$$
D=\left\{\left(x_{1}, x_{2}\right) \mid 0<x_{1}<a, 0<x_{2}<\varphi\left(x_{1}\right)\right\} .
$$

(iii) $L$ is an unstable focus lying in $D$.

(iv) All solutions with initial values lying in $D \backslash\{L\}$ have $L$ as their $\alpha$-limit sets and $\bar{\Gamma}_{1} \cup \bar{\Gamma}_{2} \cup \bar{\Gamma}$ as their $\omega$-limit sets.

Note that such a system can be constructed (see [8, pp. 405-409]).

We now define

$$
f_{i}\left(x_{1}, x_{2}\right)= \begin{cases}\left(x_{2}-\varphi\left(x_{1}\right)\right)^{2} g_{i}\left(x_{1}, x_{2}\right), & \left(x_{1}, x_{2}\right) \in \bar{D} \\ -\left(x_{2}-\varphi\left(x_{1}\right)\right)^{2}, & \left(x_{1}, x_{2}\right) \in \mathbf{R}_{+}^{2} \backslash \bar{D} .\end{cases}
$$

Then system (12) has the properties that $x_{2}=\varphi\left(x_{1}\right)$ is a curve of equilibria and solutions initiating in $\mathbf{R}_{+}^{2} \backslash \bar{D}$ approach $\bar{\Gamma}$. Hence (12) is dissipative (in fact trajectories initiating in $\stackrel{\circ}{R}_{+}^{2} \backslash D$ approach $\Gamma$ ). Trajectories initiating in $D$ have the same properties as described for system (13), and hence system (12) weakly persists, but does not persist.

Example $6(P \nRightarrow U P)$. If, in the previous example, one replaces the unstable focus $L$ by a center and all orbits in the interior of $\bar{\Gamma}_{1} \cup \bar{\Gamma}_{2} \cup \bar{\Gamma}$ are closed, then a persistent but not uniformly persistent dissipative system is obtained.

Remark 7. If dissipativity does not hold, then clearly all four definitions are independent. However, if $\partial E$ is compact, then, under the same assumptions, i.e., the flow $\partial \mathscr{F}$ is isolated and acyclic (except for dissipativity) as in [2], $W P \Leftrightarrow P \Leftrightarrow U P$ holds. The proof of this is analagous to the proof in [2].

\section{KOLMOGOROV SYSTEMS}

In this section we consider autonomous Kolmogorov systems of the form

$$
\dot{x}_{i}=x_{i} f_{i}\left(x_{1}, \ldots, x_{n}\right), \quad i=1, \ldots, n,
$$

as well as nonautonomous, periodic Kolmogorov systems

$\dot{x}_{i}=x_{i} g_{i}\left(t, x_{1}, \ldots, x_{n}\right)=x_{i} g_{i}\left(t+T, x_{1}, \ldots, x_{n}\right), \quad i=1, \ldots, n, T>0$. 
For the flow described by (14), $E=\mathbf{R}_{+}^{n}$ and $\partial E$ is invariant under this flow. Also, the definitions $W P, P, W U P, U P$ may be written in coordinate form

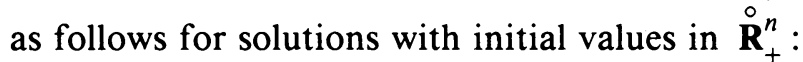

(i) (14) is $W P$ if $\limsup _{t \rightarrow \infty} \min _{1 \leq i \leq n}\left\{x_{i}(t)\right\}>0$;

(ii) (14) is $P$ if $\liminf _{t \rightarrow \infty} x_{i}(t)>0, \forall i=1, \ldots, n$;

(iii) (14) is $W U P$ if $\exists \varepsilon_{0}>0 \ni \lim \sup _{t \rightarrow \infty} \min \left\{x_{i}(t)\right\}>\varepsilon_{0}$;

(iv) (14) is $U P$ if $\exists \varepsilon_{0}>0 \ni \liminf _{t \rightarrow \infty} x_{i}(t)>\varepsilon_{0} \forall i=1, \ldots, n$.

In addition to the above, one can define for system (14) a weaker form of weak persistence, $W W P$, as follows:

(v) (14) is $W W P$ if $\lim \sup _{t \rightarrow \infty} x_{i}(t)>0 \forall i=1, \ldots, n$.

We note that in several papers in the literature (see e.g. [5]), what was defined as $W P$ was actually $W W P$.

Clearly $W P \Rightarrow W W P$. The next example shows that the reverse is not true, even if (14) is dissipative.

Example 8. Consider the nontransitive competition model described in $[15,18]$,

$$
\begin{aligned}
& \dot{x}_{1}=x_{1}\left(1-x_{1}-\alpha x_{2}-\beta x_{3}\right) \\
& \dot{x}_{2}=x_{2}\left(1-\beta x_{1}-x_{2}-\alpha x_{3}\right) \\
& \dot{x}_{3}=x_{3}\left(1-\alpha x_{1}-\beta x_{2}-x_{3}\right),
\end{aligned}
$$

where $\alpha+\beta>2, \alpha<1$. There are five equilibria, $O(0,0,0), P_{1}(1,0,0)$, $P_{2}(0,1,0), P_{3}(0,0,1), L(l, l, l)$, where $l=(1+\alpha+\beta)^{-1} . P_{i}, i=1,2,3$ are hyperbolic saddle points such that $P_{2}$ and $P_{1}$ are connected by a separatrix orbit $\Gamma_{21}$ in the $x_{1}-x_{2}$ plane and directed from $P_{2}$ to $P_{1} \cdot \Gamma_{21}$ can be parametrized by a relation of the form $x_{2}=\varphi_{21}\left(x_{1}\right), x_{3}=0,0<x_{1}<1$. Here we extend $\varphi_{21}$ in a smooth manner in $\mathbf{R}^{+}$so that $\varphi_{21}\left(x_{1}\right)<0$ for $x_{1}>1$. Similarly there exists $\Gamma_{13}$ defining $x_{1}=\varphi_{13}\left(x_{3}\right), x_{2}=0$, and $\Gamma_{32}$ defining $x_{3}=\varphi_{32}\left(x_{2}\right), x_{1}=0$. As was shown in [15], every orbit with positive initial conditions (except for two singular orbits which tend to $L$ and $L$ itself) has $\Gamma=\bar{\Gamma}_{21} \cup \bar{\Gamma}_{13} \cup \bar{\Gamma}_{32}$ as its $\omega$-limit set. Hence this is an example of a system which exhibits $W W P$, but not $W P$.

We now define a new system by multiplying the right hand sides of (16) by

$$
\left[x_{3}^{2}+\left(x_{2}-\varphi_{21}\left(x_{1}\right)\right)^{2}\right]\left[x_{2}^{2}+\left(x_{1}-\varphi_{13}\left(x_{3}\right)\right)^{2}\right]\left[x_{1}^{2}+\left(x_{3}-\varphi_{32}\left(x_{2}\right)\right)^{2}\right] \text {. }
$$

For this new system all points lying on $\Gamma$ are equilibria. All other orbits together with their orientations are the same as for orbits of (16). We note that for this example, the system remains $W W P$, but not $W P$, and all the conditions of the main theorem of [2], i.e., dissipativity, isolatedness, acyclicity except $W P$ (replaced by $W W P$ ) are satisfied. The points of $\Gamma$ were fixed to obtain the acyclic condition. So, from $W W P$ under these conditions, it does not follow that $U P \Leftrightarrow P \Leftrightarrow W U P \Leftrightarrow W P$.

Finally, we consider system (15). This system may be transformed into an autonomous system (but not of Kolmogorov type) by introducing the coordinate 
$\theta=t$. Then we have the transformed system

$$
\begin{aligned}
x_{i}^{\prime} & =x_{i} f_{i}\left(\theta, x_{1}, \ldots, x_{n}\right), \quad i=1, \ldots, n \\
\theta^{\prime} & =1,
\end{aligned}
$$

which defines a flow on the cylindrical phase space $E=S^{1} \times \mathbf{R}_{+}^{n}$, where points $\left(\theta, x_{1}, \ldots, x_{n}\right)$ and $\left(\theta+T, x_{1}, \ldots, x_{n}\right)$ coincide. Here $\partial E=S^{1} \times \partial \mathbf{R}_{+}^{n}$. The persistence definitions are the same as for system (14) (there is no condition for $\theta$ ). We note that in this case dissipativity follows whenever there exists a $\rho>0$ such that, for all solutions, $\lim \sup _{t \rightarrow \infty}\|x(t)\|<\rho$ (see [16]).

\section{ACKNOWLEDGMENT}

We thank the referee for some useful remarks especially concerning $\S 4$.

\section{REFERENCES}

1. T. Burton and V. Hutson, Repellers in systems with infinite delay, J. Math. Anal. Appl. 137 (1989), 240-263.

2. G. Butler, H. I. Freedman and P. Waltman, Uniformly persistent systems, Proc. Amer. Math. Soc. 96 (1986), 425-429.

3. __ Persistence in dynamical systems, J. Differential Equations 63 (1986), 255-263.

4. A. Fonda, Uniformly persistent semidynamical systems, Proc. Amer. Math. Soc. 104 (1988), 111-116.

5. H. I. Freedman and P. Waltman, Persistence in models of three interacting predator-prey populations, Math. Biosci. 68 (1984), 213-231.

6. __ Persistence in a model of three competitive populations, Math. Biosci. 73 (1985), 89101.

7. T. C. Gard, Uniform persistence in multispecies population models, Math. Biosci. 85 (1987), 93-104.

8. J. Guckenheimer and $\mathrm{Ph}$. Holmes, Nonlinear oscillations, dynamical systems, and bifurcation of vector fields, Applied Math. Sci. 42 (1983).

9. J. K. Hale and P. Waltman, Persistence in infinite-dimensional systems, SIAM J. Math. Anal. 20 (1989), 388-395.

10. J. Hofbauer and K. Sigmund, Permanence for replicator equations, Lecture Notes in Economics and Math. Systems, vol. 287, Springer (1987), 70-85.

11. V. Hutson, $A$ theorem on average Liapunov functions, Monatsh. Math. 98 (1984), 267-275.

12. V. Hutson and R. Law, Permanent coexistence in general models of three interacting species, J. Math. Biol. 21 (1985), 285-298.

13. V. Hutson and K. Schmitt, Permanence in dynamical systems, preprint.

14. G. Kirlinger, Permanence in Lotka-Volterra equations: Linked predator-prey systems, Math. Biosci. 82 (1986), 165-191.

15. R. M. May and W. J. Leonard, Nonlinear aspects of competition between three species, SIAM J. Appl. Math. 29 (1975), 243-253.

16. V. A. Pliss, Nonlocal problems of the theory of oscillations, Academic Press, New York, 1966.

17. H. Rouche, P. Habets and M. Laloy, Stability theory by Liapunov's direct method, Appl. Math. Sci. 22 (1977). 
18. P. Schuster, K. Sigmund and R. Wolff, On $\omega$-limits for competition between three species, SIAM J. Appl. Math. 37 (1979), 49-54.

19. J. Hofbauer and J. W-H. So, Uniform persistence and repellers for maps, Proc. Amer. Math. Soc. 107 (1989), 1137-1142.

Department of Mathematics, University of Alberta, Edmonton, Alberta T6G 2G1 CANADA

Department of Mathematics, Technical University of Budapest, Budapest, Hungary, H-1521 